\title{
REVIEW
}

\section{Posthaemorrhagic ventricular dilatation}

\section{A Whitelaw, M Thoresen, I Pople}

\section{Posthaemorrhagic ventricular dilatation is the most} serious direct complication of intraventricular haemorrhage after preterm birth. It results initially from multiple small blood clots throughout the cerebrospinal fluid channels impeding circulation and reabsorption. Management is difficult and new treatment approaches are needed.

See end of article for authors' affiliations

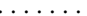

Correspondence to: Professor Whitelaw Division of Child Health, University of Bristol Medical School, Southmead Hospital, Bristo BS9 1PJ, UK. andrew.whitelaw@ bristol.ac.uk
$\mathrm{D}$ spite the reduction in the proportion of small preterm infants who develop intraventricular haemorrhage (IVH), the increase in survival of the most immature infants means that there are now more infants who are candidates to develop IVH than 20 years ago. The most severe direct complication of IVH is the progressive accumulation of cerebrospinal fluid under pressure with ballooning of the ventricles and increasing enlargement of both the ventricular system and the head.

\section{DEFINITION}

Posthaemorrhagic ventricular dilatation (PHVD) precedes hydrocephalus but may be self limiting and is widely defined as IVH followed by progressive enlargement until the ventricular width at the intraventricular foramen exceeds $4 \mathrm{~mm}$ over the 97th centile for gestational age ${ }^{1}$ (fig 1). Infants who fulfil these criteria have a serious prognosis, with about $50-60 \%$ being shunt dependent, over $60 \%$ disabled, and around $20 \%$ dying. ${ }^{2}$ Hydrocephalus usually refers to infants with progressive enlargement of the head and the ventricular system.

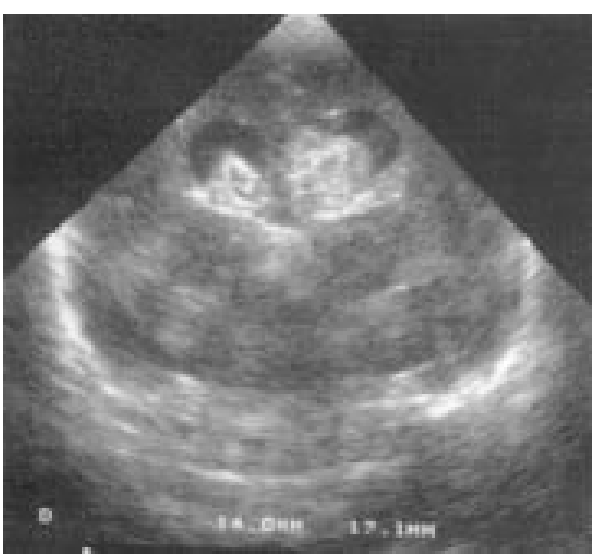

Figure 1 Cranial ultrasound (coronal view) showing enlarged ballooned ventricles (black) with blood clot (white) in the lumina. The ventricular width is measured from the midline to the lateral border.

\section{DISTINGUISHING VENTRICULAR ENLARGEMENT DRIVEN BY CEREBROSPINAL FLUID (CSF) FROM CEREBRAL ATROPHY}

It is important to distinguish ventricular enlargement due to atrophy (when the ventricles are not round, enlargement is slow, and head enlargement is not increased) from ventricular dilatation due to excessive build up of CSF (when the ventricles are usually round (fig 1), enlargement may be rapid, and increased head enlargement follows in time).

\section{MECHANISMS BEHIND} POSTHAEMORRHAGIC HYDROCEPHALUS

The traditional explanation is that CSF is reabsorbed through arachnoid villi which project into the blood filled cranial venous sinuses, but it is also likely that it is reabsorbed across the ependyma into small blood vessels within the central nervous system. ${ }^{4}$ Such small channels and the foramina can be blocked by multiple small blood clots. There is some endogenous attempt to lyse blood clots in the CSF but such fibrinolysis is relatively inefficient, ${ }^{5}$ probably because the concentrations of plasminogen are low and there are high concentrations of an inhibitor of plasminogen activator. ${ }^{67}$ Transforming growth factor $\beta 1$ (TGF $\beta 1$ ) is released into the CSF after IVH. It has the biological property of upregulating the genes for extracellular matrix proteins such as fibronectin and laminin and is involved in wound healing, scar formation, and fibrotic diseases such as cirrhosis, fibrosing alveolitis, and diabetic nephropathy.

TGF $\beta 1$ is virtually undetectable in normal neonatal CSF, but is increased in CSF from infants with PHVD (fig 2). CSF TGF 31 is even higher in infants who later develop permanent hydrocephalus. $^{8}$ Prolonged elevation of CSF TGF $\beta 1$ levels in one infant was followed by later shunt dependence, whereas initially raised levels that normalised were seen in one infant who did not need a shunt. Further evidence for the role of TFG $\beta 1$ in hydrocephalus is provided by transgenic mice that overexpress TGF $\beta 1$ in the central nervous system. Virtually all such mice developed obvious hydrocephalus. ${ }^{9}$ The origin of the TGF $\beta 1$ in the CSF could be from the haemorrhage itself (because TGF $\beta 1$ is stored in platelets) or from the choroid plexus.

Abbreviations: IVH, intraventricular haemorrhage; PHVD, posthaemorrhagic ventricular dilatation; CSF cerebrospinal fluid; TGF $\beta 1$, transforming growth factor $\beta 1$ 


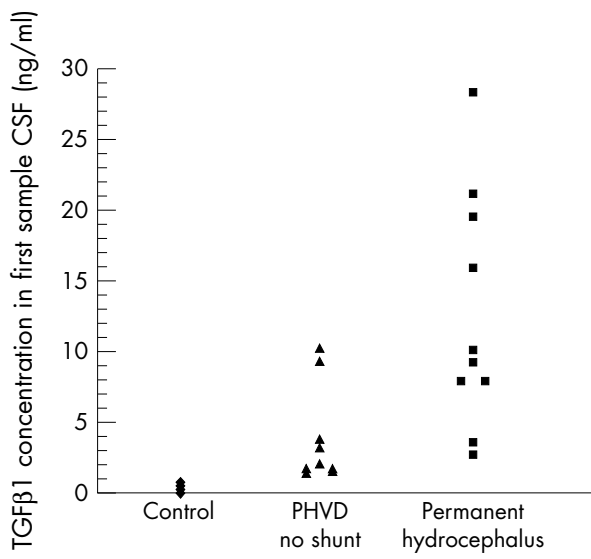

Figure 2 Concentrations of transforming growth factor $\beta 1$ (TGF $\beta 1$ ) in the cerebrospinal fluid (CSF; first sample) of control infants and infants with posthaemorrhagic ventricular dilatation (PHVD). From Whitelaw et a ${ }^{\beta}$ with permission.

\section{CURRENT HYPOTHESIS ON THE PATHOGENESIS OF PHVD}

The current concept is that multiple small blood clots throughout the CSF partially occlude the channels of circulation and reabsorption, and TGF $\beta 1$ is released into the CSF. Some ventricular dilatation may occur early, but this is potentially reversible if the blood clots are lysed quickly. Over a period of two to six weeks, TGF $\beta 1$ stimulates the production of extracellular matrix proteins around the brain stem and in the subarachnoid space. If this deposition is extensive, sufficient "scar tissue" may be created to effectively block the exits from the fourth ventricle (creating obstructive hydrocephalus) or the channels of reabsorption (giving communicating hydrocephalus). If an effective block occurs, CSF accumulates under pressure, and progressive ventricular dilatation occurs. Periventricular white matter may then be damaged because of raised pressure and oedema, ${ }^{10}$ free radical damage from iron, ${ }^{11}$ or proinflammatory cytokines. ${ }^{12}$

\section{MANAGEMENT OF PHVD Initial assessment}

(1) Diagnosis using recognised criteria and measurement of ventricular width.

(2) Ascertainment of parenchymal brain lesions that may affect the infant's prognosis.

(3) Clinical assessment including neurological status (irritability, increased tendon reflexes, squamosal suture $>5 \mathrm{~mm}$ ), head circumference increasing $>7 \mathrm{~mm} /$ week, and tension of fontanelles.

\section{Interventions}

There are two objectives when treating infants with PHVD:

(a) to protect the infant from damage secondary to raised intracranial pressure;

(b) to avoid the need for a permanent shunt with the long term complications of blockage and infection necessitating multiple revisions.

We see no point in treating infants who have had an IVH and moderate dilatation of the ventricles with the ventricular width less than $4 \mathrm{~mm}$ above the 97th centile.

If the ventricles gradually enlarge beyond the 97 th centile + $4 \mathrm{~mm}$ limit, then the infant meets the entry criteria used in most of the therapeutic trials.

Early and repeated lumbar punctures or ventricular taps Early and repeated lumbar punctures or ventricular taps have been evaluated in four randomised trials. ${ }^{13}$ In total, 280 infants were recruited, and this intervention made no difference to the numbers of shunt operations, deaths, or disabled infants. There was a trend towards increased CSF infection in infants who had multiple taps.

\section{Diuretic therapy}

Acetazolamide and frusemide reduce the production of CSF by about half and appeared to offer a non-invasive oral treatment that might prevent the need for shunt surgery. Two controlled trials involving 193 infants have been systematically reviewed.$^{14}$ Diuretic therapy significantly increased the risk of impairment or disability at 1 year (relative risk 1.27 (95\% confidence interval (CI) 1.02 to 1.58 ) and nephrocalcinosis (relative risk 5.31 (95\% CI 1.90 to 14.8$)$ ), with no decrease in death or ventriculoperitoneal shunt. The lack of therapeutic effect underlines the fact that PHVD is not due to overproduction of CSF. The worsening of outcome may be due to the effect of acetazolamide on $\mathrm{CO}_{2}$ elimination or acid-base or electrolyte balance, or it may indicate that carbonic anhydrase is necessary for maturation of normal white matter. ${ }^{15}$

\section{Intraventricular fibrinolytic treatment}

Two animal model studies have shown that injection of urokinase or tissue plasminogen activator can prevent hydrocephalus after the injection of blood into the ventricles or cisterna magna. ${ }^{16}{ }^{17}$ This led to trials of intraventricular injection of fibrinolytic agents in infants with PHVD. A small number of uncontrolled pilot studies have given results ranging from $100 \%$ success to $100 \%$ failure, and the only randomised trial showed that $50 \%$ of the infants needed a shunt in the streptokinase and the control group. ${ }^{18}$ This intervention has a significant risk of infection of the central nervous system and also secondary bleeding. The clinical trials of intraventricular fibrinolytic treatment started several weeks after the IVH, whereas in the two animal model studies the intervention was given within 24 hours of the injection of blood. Furthermore, it has been shown that intraventricular injection of tissue plasminogen activator consistently increases the concentration of TGF $\beta 1$ in the CSF, presumably enhancing the process leading to hydrocephalus. ${ }^{8}$

\section{Surgical interventions for PHVD}

External ventricular drainage allows immediate control of raised intracranial pressure and removes blood and protein. The major problem is the high risk of CSF infection, which increases with the duration of drainage. As CSF infection is, in itself, a major risk factor for shunt dependence and disability, external ventricular drainage is generally reserved for cases in which there is bloody CSF with symptomatic raised intracranial pressure which cannot be lowered in any other way. Blockage of the catheter by blood clots is a common problem.

Subcutaneous placement of a ventricular reservoir allows easy drainage of CSF as often as is required to normalise CSF pressure and ventricular enlargement. It requires the availability of a neurosurgeon, and the size of the reservoir carries a risk of skin ulceration in the smallest infants. There is also a significant risk of infection.

Another surgical technique that has been used in infants with slowly progressing, communicating hydrocephalus is endoscopic coagulation of the choroid plexus, which is the main source of CSF. Since 1973, 125 of these procedures have been performed in Bristol. About two thirds of infants with such slowly progressing posthaemorrhagic hydrocephalus were stabilised by this technique, and the significant complications of a long term CSF shunt were avoided. ${ }^{19}$ Third ventriculostomy is another procedure that can be carried out endoscopically and can relieve obstructive hydrocephalus in infants. However, in a series of 10 premature infants with posthaemorrhagic hydrocephalus seven were failures. ${ }^{20}$

Ventriculoperitoneal shunt is the traditional treatment of most forms of hydrocephalus, but this is not an option early 
after IVH in small preterm infants because of the extremely high risk of blockage, infection, and skin ulceration. There have been no controlled trials of surgical treatments of PHVD.

\section{Management of PHVD without symptomatic raised} pressure

The current management of PHVD in the absence of symptomatic raised intracranial pressure is generally to observe the infant and avoid the considerable iatrogenic complications from all of the above interventions. If ventricular and head enlargement progress relentlessly and the CSF protein has fallen below $1.5 \mathrm{~g} / \mathrm{l}$, then ventriculoperitoneal shunt surgery can be planned electively.

Management of PHVD with symptomatic raised pressure If the infant develops rapid dilatation of the ventricles with a large amount of blood clot visible on ultrasound and rapid head enlargement, it is likely that there is obstructive hydrocephalus and raised pressure. The upper limit of normal CSF pressure is $6 \mathrm{~mm} \mathrm{Hg}$, and we consider CSF pressures of 12 $\mathrm{mm} \mathrm{Hg}$ or higher to be likely to be harmful because of the frequency of adverse changes in cerebral blood flow velocity, evoked potentials, and reversible neurological signs at such pressures. If the clinician concludes that the infant with PHVD has raised pressure, it is imperative that the pressure be lowered. Lumbar puncture is generally not effective in draining off enough fluid (>10 ml/kg), and ventricular puncture is usually necessary. This should be carried out with great attention to sterile technique, entering the anterior fontanelle $1.0-1.5 \mathrm{~cm}$ lateral to the midline. The depth of insertion required can be estimated on ultrasound beforehand. At least $10 \mathrm{ml} / \mathrm{kg}$ should be removed, allowing the fluid to drip spontaneously. No more than $20 \mathrm{ml} / \mathrm{kg}$ should be taken at any one session, as removal of such volumes within a few minutes is likely to produce midline shift, with destabilisation, bradycardia, and desaturation.

\section{Experimental interventions for PHVD}

A radical new intervention with the objectives of $(a)$ removing intraventricular blood, (b) removing TGF $\beta,(c)$ normalising intracranial pressure, and $(d)$ removing non-protein-bound iron and proinflammatory cytokines has been piloted in Bristol. The treatment criteria were the same as for the other treatment trials (IVH and ventricular width $4 \mathrm{~mm}$ over the 97th centile), and we suspect, on the basis of limited evidence and the progressive nature of the pathology, that 3 weeks of age may be the maximum effective age limit. The procedure is to insert a ventricular catheter into the right ventricle frontally and another catheter into the left ventricle posteriorly. Recombinant tissue plasminogen activator $(0.5 \mathrm{mg} / \mathrm{kg})$ is injected into the right ventricle and left for eight hours. Artificial cerebrospinal fluid is then infused at $20 \mathrm{ml} / \mathrm{kg} /$ hour into the right ventricle, and old blood and debris are drained from the left ventricular drain to keep the intracranial pressure below $7 \mathrm{~mm} \mathrm{Hg}$. Irrigation is continued until the draining fluid is clear of old blood, usually in 72 hours but sometimes taking up to a week. The combination drainage, irrigation, and fibrinolytic treatment has been given the acronym DRIFT.
This highly invasive intervention is still at the pilot stage, is demanding of nursing resources, and clearly has the potential to make matters worse if scrupulous care is not taken at every surgical, medical, and nursing level.

Future interventions aimed at preventing hydrocephalus at a molecular level are being planned in laboratories, but we are years away from obtaining the necessary regulatory clearance to start clinical trials.

$\ldots \ldots \ldots \ldots \ldots, \ldots$

Authors' affiliations

A Whitelaw, M Thoresen, Division of Child Health, University of Bristol, Bristol, UK

I Pople, Department of Neurosurgery, Frenchay Hospital, Bristol, UK

\section{REFERENCES}

1 Levene MI. Measurement of the growth of the lateral ventricles in preterm infants with real time ultrasound. Arch Dis Child 1981:56:900-4.

2 Ventriculomegaly Trial Group. Randomised trial of early tapping in neonatal posthaemorrhagic ventricular dilatation. Arch Dis Child 1990;65:3-10

3 International PHVD Drug Trial Group. International randomised trial of acetazolamide and frusemide in posthaemorrhagic ventricular dilatation. Lancet 1998:352:433-40.

4 Whitelaw A. We need a new understanding of the circulation of cerebrospinal fluid. Acta Paediatr 1997;86:133-4.

5 Whitelaw A. Endogenous fibrinolysis in neonatal cerebrospinal fluid. Eur J Pediatr 1993;152:928-30.

6 Whitelaw A, Mowinckel MC, Abildgaard U. Low levels of plasminogen in cerebrospinal fluid after intraventricular haemorrhage: a limiting factor for clot lysis? Acta Paediatr 1995;84:933-6

7 Hansen A, Whitelaw A, Lapp C, et al. Cerebrospinal fluid plasminogen activator inhibitor-1: a prognostic factor in posthaemorrhagic hydrocephalus. Acta Paediatr 1997:86:995-8.

8 Whitelaw A, Christie S, Pople I. Transforming growth factor $\beta 1$ : a possible signal molecule for post-haemorrhagic hydrocephalus? Pediatr Res 1999;46:576-80

9 Galbreath E, Kim SJ, Park K, et al. Overexpression of TGF $\beta 1$ in the central nervous system of transgenic mice results in hydrocephalus. $J$ Neuropathol Exp Neurol 1995:54:339-49.

10 Kaiser A, Whitelaw A. Cerebrospinal fluid pressure during post-haemorrhagic ventricular dilatation in newborn infants. Arch Dis Child 1985;60:920-3.

11 Savman K, Nilsson U, Blennow M, et al. Non-protein-bound iron in cerebrospinal fluid from preterm infants with posthemorrhagic ventricular dilatation. Pediatr Res 2001:49:208-12.

12 Savman K, Blennow M, Hagberg H, et al. Cytokine response in cerebrospinal fluid from preterm infants with posthemorrhagic ventricular dilatation [Abstract]. Pediatr Res 2001;49:365A.

13 Whitelaw A. Repeated lumbar punctures or ventricular taps for preventing morbidity and shunt dependence in newborn infants with intraventricular haemorrhage. In: The Cochrane Library, Issue 1. Oxford: Update Software, 2000.

14 Whitelaw A, Kennedy C, Brion L. Diuretic therapy for newborn infants with post-hemorrhagic ventricular dilatation. In: The Cochrane Library, Issue 2. Oxford: Update Software, 2001.

15 Cammer WB, Brion LP. Carbonic anhydrase in nervous tissue. In: Chegwidden WR, Carter ND, Edwards YH, eds. The carbonic anhydrases: new horizons. Basel: Birkhauser Verlag, 2000:475-89.

16 Pang D, Sclabassi RJ, Horton JA. Lysis of intraventricular blood clot with urokinase in a canine model: part 3. Neurosurgery 1986;19:553-72.

17 Brinker T, Seifert V, Dietz H. Subacute hydrocephalus after experimental subarachnoid hemorrhage: its prevention by intrathecal fibrinolysis with recombinant tissue plasminogen activator. Neurosurgery 1992;31:306-11.

18 Whitelaw A. Intraventricular streptokinase after intraventricular hemorrhage. In: The Cochrane Library, Issue 1. Oxford: Update Software, 2000

19 Pople IK, Ettles D. The role of endoscopic choroid plexus coagulation in the management of hydrocephalus. Neurosurgery 1995:36;698-701.

20 Buxton N, Macarthur D, Malluci C, et al. Neuroendoscopy in the premature population. Childs Nerv Syst 1998;14:649-52. 